\title{
Case report: A rare case of dysgerminoma presenting with skin and breast metastasis
}

\author{
Oguntayo O. Adekunle ${ }^{1^{*}}$, Marliya Zayyan ${ }^{1}$, Abimbola O. D. Kolawole ${ }^{1}$, Saa'd Ahmed ${ }^{2}$
}

${ }^{1}$ Department of O \& G, Ahmadu Bello University Teaching Hospital Complex, Shika, Zaria;

*Corresponding Author: fayokunmi@yahoo.co.uk

${ }^{2}$ Department of Pathology, Ahmadu Bello University Teaching Hospital Complex, Shika, Zaria

Received 22 January 2013; revised 1 April 2013; accepted 14 April 2013

Copyright (C) 2013 Oguntayo O. Adekunle et al. This is an open access article distributed under the Creative Commons Attribution License, which permits unrestricted use, distribution, and reproduction in any medium, provided the original work is properly cited.

\begin{abstract}
A 30 years old grandmultiparous woman presented with 6 month history of severe abdominal pain and 4 month history of progressive abdominal swelling associated with multiple swellings on her abdomen and her breasts. She had laparotomy two years earlier for a suspected fibroid. A repeat laparatomy discovered a huge left ovarian tumour; which are multilobulated and hard. She had Total Abdominal Hysterectomy and Salpingectomy with Lumpectomy on both breast. Histology report turned out as Dysgerminoma, breast, Subcutaneous Tissue and Skin Metastatic Dysgerminoma.
\end{abstract}

Keywords: Dysgerminoma; Metastasis;

Subcutaneous and Breast

\section{CASE REPORT}

Mrs. DH was a 30 years old Para6 $6^{+3}, 5$ children alive presenting with a 6-month history of severe abdominal pain and a progressive abdominal swelling of 4 months duration following her last child birth six months earlier. She also noticed some other smaller swellings on the anterior abdominal wall and both breasts. See Figures 1 and 2. She had abdominal pain which was severe enough to prevent her from doing her normal chores at home. It was localized to her suprapubic region and constant.

She has no menstrual abnormality. She had never used any form of contraceptive method. All her pregnancies were spontaneously conceived; she had spontaneous miscarriages in her 1st, 4th and 8th pregnancies respectively. The 6th child was an early neonatal death due to a febrile illness. All other children are alive and well.

She had laparatomy at the referring hospital two years earlier for uterine fibroid.
On examination she was a young ill-looking woman. She was pink and well hydrated. There was no significant, palpable peripheral lymph nodes.

There were solitary mass in each breast. Each measured about $8 \times 4 \mathrm{~cm}$ (See Figure 2). They were both fixed to the skin but free from the underlying structures

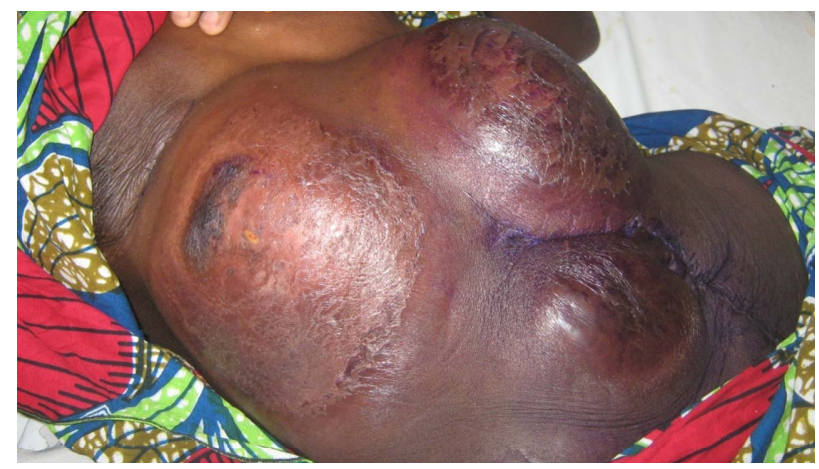

Figure 1. Tumour metastasis to the anterior abdominal wall skin and subcutaneous tissues.

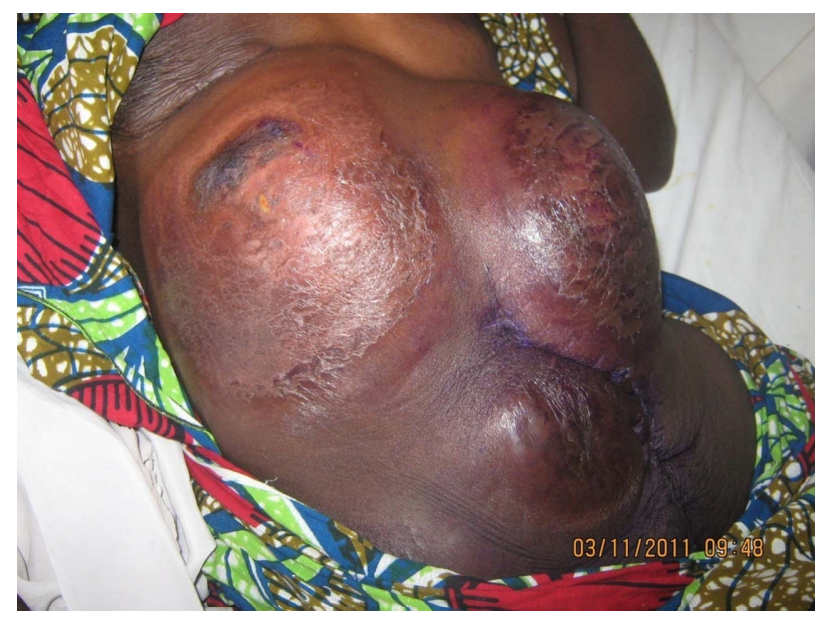

Figure 2. Tumour is evident on the left breast and the anterior abdominal wall. 
and were not-tender.

Her cardio-respiratory systems were normal.

The abdomen was enlarged and moved with respiration. There were multiple discrete masses on the anterior abdominal wall, the largest measuring about $12 \times 8 \mathrm{~cm}$ while the smallest measured $4 \times 4 \mathrm{~cm}$ (see Figure 1); they were attached to the anterior abdominal wall, tender and hard. There was a supra-pubic mass that was consistent with a 24 weeks gravid uterus. The mass was mobile from side to side but tender. It was not attached to the over lying structures of the anterior abdominal wall. Ascites was not demonstrable because of the anterior abdominal wall masses.

She had a normal vulva and vagina. The cervix was grossly normal. The uterus was normal in size and separate from the mass. There was no abnormality detected on rectal examination.

A diagnosis of ovarian tumour with metastasis to the skin \& breast was made.

Ultrasound scan made a diagnosis of ovarian tumour with subcutaneous metastasis.

At laparatomy a huge left ovarian tumour; multilobulated, hard with bowels and omental adhesion. She had Total Abdominal Hysterectomy and Salpingectomy with Lumpectomy on both breast.

Histology report turned out as Dysgerminoma. Breast, Subcutaneous tissue and Skin Metastatic Dysgerminoma (See Figures 3 and 4).

\section{DISCUSSION}

The 3 major types of ovarian tumors are epithelial, sex cord, and germ cell. Epithelial cell tumors represent the majority of all ovarian neoplasm's (82\%). Conversely, germ cell tumors (GCTs) are rare, comprising approximately $20 \%$ of all ovarian tumors, both benign and malignant [1].

A dysgerminoma is a tumor of the ovary that is composed of primitive, undifferentiated germ cells [2]. Germ cell tumors arise from primordial germ cells of the ovary and the testis. Of these ovarian lesions, 97\% are benign proliferations (i.e. mature teratomas); the remaining 3\% are malignant [2].

This is a rare case as only $3 \%$ dysgerminomas are malignant, although it is still the most common malignant germ cell tumour occurring in the ovary. The Germ cell Tumors have endocrine activities, as such they can be worked up or tracked using the following tumor markers beta human chorionic gonadotropin (bHCG), alpha-fetoprotein (AFP), lactate dehydrogenase (LDH), caner antigen 125 (CA125). These markers also can be use for postoperative follow-up or tracking the success of adjuvant therapy. Dysgerminomas (a germ cell subtype) are exception to the endocrine activities, therefore absence of elevated bHCG and /or AFP does not exclude dysger-

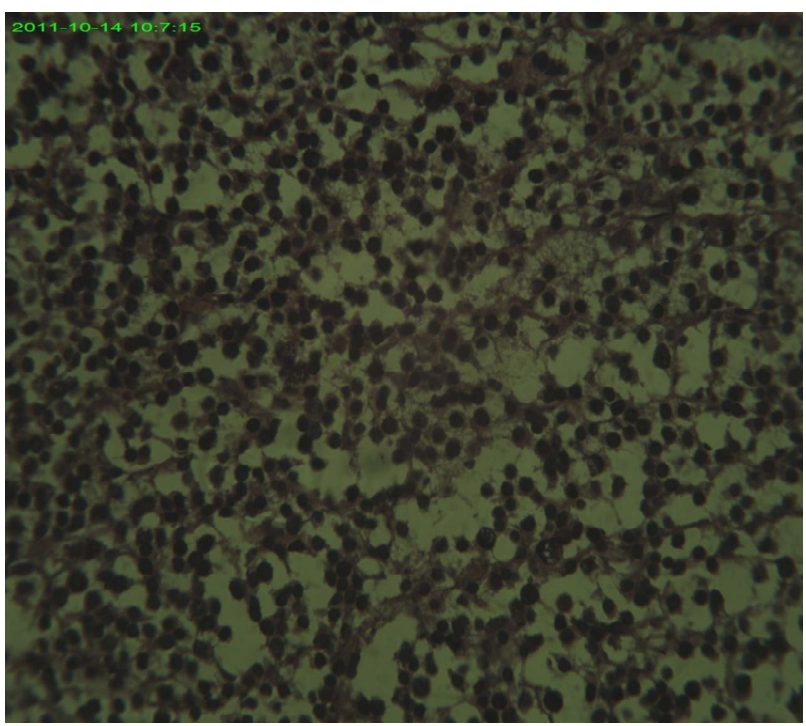

Figure 3. Note: Tumour cells has hyperchromatic nucle and clear cytoplasm, these are separated by thin fibrous strands containing lymphocytes. $\mathrm{H} \& \mathrm{E} \times 400$.

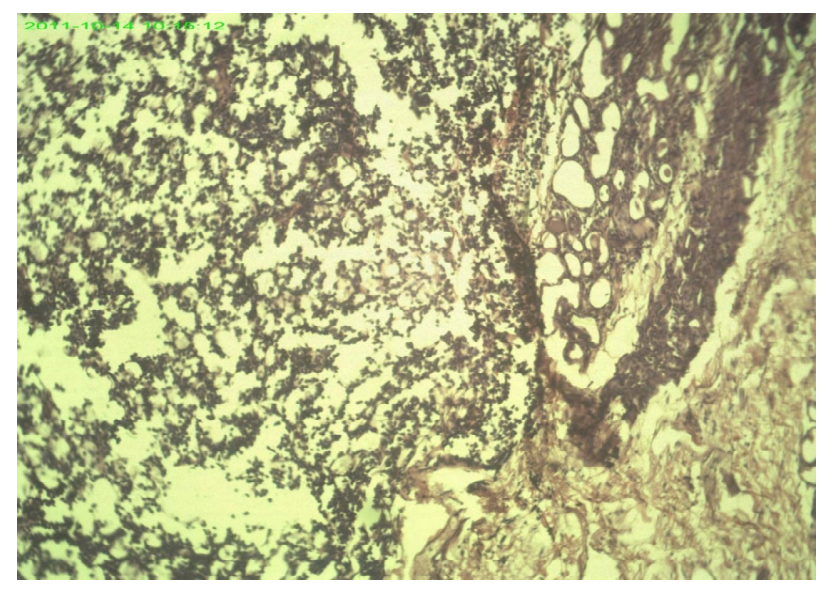

Figure 4. Note: Infiltration of breast lobule by tumour H \& E $\times 40$.

minomas. The use of bHCG and AFP assay are highly recommended. [3]

Mrs. D.H. did not only present with a dysgerminoma but a rare component of dysgerminoma. She also had metastasis to the breast, skin and subcutaneous tissue that has only been reported once in the breast in the literature in a 16 years old girl [4]. Extra ovarian tumor spread of dysgerminomas often involves the retroperitoneal and pelvic lymph nodes; hematogenous spread may occur; common sites of involvement are the lungs, liver, and bone [3].

These lesions are found most commonly in adolescents $(60 \%$ of cases are diagnosed in patients less than 20 years) and young adults [5].

Dysgerminomas are characterized by their solid nature and rapid growth. They often measure more than $10 \mathrm{~cm}$ 
in maximum dimension at the time of diagnosis [5].

Unlike other germ cell tumors, dysgerminomas often occur bilaterally (approximately $10 \%$ - 20\% of cases). In this case it was unilateral and it was on the left side [5].

Common signs and symptoms include abdominal/pelvic pain (55\% - 85\%), abdominal mass (35\%), fever (10\% - 25\%), vaginal bleeding (10\%), and, occasionally, ascites. Mrs. D.H. had a similar presentation excluding fever, vaginal bleeding and ascites [1,5].

The main differential in the differential diagnosis of dysgerminoma is lymphoma/leukemia, when we consider the mode of presentation. Dysgerminoma of the ovary may be confused with malignant lymphoma of the ovary as it mimics lymphoma both microscopically and macroscopically [6]. Other differentials include, yolk sac tumor, embryonal carcinoma, and Sertoli cell tumors, all this are actually excluded by the histopathologist [6].

The overwhelming majority (approximately 75\%) of these tumors are limited to one or both ovaries (International Federation of Gynecologists and Obstetricians [FIGO] stage 1) at the time of diagnosis. This was not the case in our patient, she already has metastasis. This is not surprising as most of our oncology patients present late, commonly at stage 3 [5].

Patients with stage 1A disease (i.e., disease that is limited to 1 ovary) may be treated by unilateral oophorectomy alone, especially when fertility is to be preserved. The relapse rate ranges from $10 \%$ to $20 \%$; the overall survival rate is $90 \%$ - $100 \%$ [7]. Patients who suffer relapses may undergo chemotherapy; the survival rate for such patients is greater than $90 \%$ [1].

The treatment options range from simple unilateral oophorectomy alone to chemotherapy or radiotherapy in advance cases. Mrs. D.H. actually did well on surgery while awaiting the commencement of adjuvant chemotherapy. Most cases surgery alone is not adequate for treatment, the patients usually benefits from adjuvant therapies to prevent relapse [8].

Unfortunately we lost our patient to follow up, which is a usual occurrence in developing nations like ours, once they have a favorable response to treatment they rarely come back.

When chemotherapy is use $3-4$ cycles of adjuvant chemotherapy with cisplatinum, etoposide/vinblastine, and bleomycin are often reserved for patients with at least stage $1 \mathrm{~B}$ disease (i.e., disease that is limited to both ovaries) or for those patients who suffer recurrences and it has been shown that the results of therapy are similar to results for patients with stage $1 \mathrm{~A}$ disease [8].

The prognosis and treatment of dysgerminomas are associated with their pathologic and clinical stage.

\section{CONCLUSION}

A dysgerminoma is a germ cell tumor of the ovary which is rare and in this case presenting with subcutaneous metastasis, skin and the breast. This was a rare presentation involving all the tissue components. The tumour has a good prognosis at early presentation, otherwise adjuvant chemotherapy is usually needed in advance cases.

\section{REFERENCES}

[1] Michener, C.M. and Huh, W.K. (2012) Ovarian dysgerminomas. Medscape Drugs, Diseases \& Procedures.

[2] Prat, J. (2004) Germ cell tumors. Pathology of the Ovary. Saunders, Philadelphia, 251-282.

[3] Ovarian dysgerminomas (2012) http://www1.cgmh.org.tw/intr5/c6700/obgyn/f/web/Dysg erminoma/index.htm.2Dec

[4] Kattan, J., Droz, J.P., Charpentier, P., Michel, G., Lhommé, C., Boutan-Laroze, A. and Prade, M. (1992) Ovarian dysgerminoma metastatic to the breast. Gynecologic Oncology, 46, 104-106. doi:10.1016/0090-8258(92)90204-V

[5] Kurman, R.J. and Talerman, A. (2002) Germ cell tumors of the ovary. Blaustein's pathology of the female genital tract. 5th Edition, Springer-Verlag, New York, 967-1034.

[6] Dantkale, S.S., Pandit, G.A., Joshi, S.S. and Pudale, S.S. (2012) Primary bilateral non hodgins ovarian lymphoma-A case Report. Journal of Krishna Institute of Medical Sciences University, 1, 155.

[7] Pectasides, D., Pectasides, E. and Kassanos, D. (2008) Germ cell tumors of the ovary. Cancer Treatment Reviews, 34, 427-441.

[8] Guillem, V. and Poveda, A. (2007) Germ cell tumours of the ovary. Clinical and Translational Oncology, 9, 237243. 